\title{
Semantic 3D Building Model Construction for Smart Urban Management
}

\author{
Sun Xuan \\ Nankai University, Zhou Enlai School of Government, Weijin Road 94, Tianjin, China
}

\begin{abstract}
Semantic 3D building models are the basic data of various applications for smart urban management. However, it is still a challenge to produce semantic models for all the buildings in a specified large-scale area through a stable and reliable process. In this paper, a general and effective scheme is introduced for semantic 3D building model construction and some example applications are illustrated to demonstrate the great capability of semantic 3D building models in different aspects.
\end{abstract}

KEYWORD: Semantic modeling; 3D building model; Urban management; Smart city

\section{INTRODUCTION}

With the promotion of management modernization and national urbanization process, smart city construction has become one of major municipal projects of a lot of cities. As the core platform of digitalized management of city, virtual geographical environment is the basis of various smart applications. Whereas, in order to effectively analyze and utilize the information of land use status, housing situation, and population distribution pattern of cities, it is very necessary to bring in the semantic models that conform to human recognition habit, aside from the traditional geometric models which serve for visualization purpose only.

As one of the major components of digitized spatial environment of city, building model plays an important role in almost all kinds of urban GIS (geographic information systems). To explore further applications in urban planning and management, the construction of semantic 3D building model has attracted a lot of attentions from the fields of geographic science and public management.

\section{BACKGROUND}

In the related fields, there're two kinds of commonly used semantic 3D building models. One is BIM (Building Information Model), which is a comprehensive model covering all the design information throughout the whole life circle of architecture engineering (Cerovsek 2011, Isikdag et al. 2013), and the other is CityGML (Kolbe et al.
2005, 2009, Gröger \& Plümer 2012), which is a multi-level all-embracing data exchange model defined by OGC (Open GIS Consortium).

It is convenient to obtain all the detailed semantic information needed from BIM (Benner et al. 2005, $\mathrm{Wu} \&$ Hsieh 2007, Isikdag \& Zlatanova 2009). However, there are a great number of old buildings without completed design information and most of architecture design firms tend to keep their works secret. It actually faces great difficulties to integrate BIM into GIS or other information management system for smart applications.

Compared with BIM, CityGML is much more widely used in the field of GIS, but the construction of the semantic model always involves a great deal of manual operation (Kelly \& Wonka 2011, Krecklau \& Kobbelt 2012). Although some scholars proposed to generate semantic 3D building models with the data collected from open source map on the web, like OpenStreetMap (Goetz \& Zipf 2012, 2013), it is still a challenge to produce semantic models for all the buildings in a specified large-scale area through a stable and reliable process.

\section{METHOD}

The semantic model can be recognized as the combination of conceptual model and coherently related geometric model. And the construction procedure of semantic 3D building model is divided into the following three steps. 


\subsection{Geometric modeling}

Rapid development of survey and mapping technology makes it much easier to generate highresolution models in a large-scale area. A lot of cities and districts have been working on digital city construction, where air-borne and mobile Lidar (Light detection and ranging) devices are employed to collect accurate positions of every part of urban objects and construct detailed 3D models (see Fig.1).
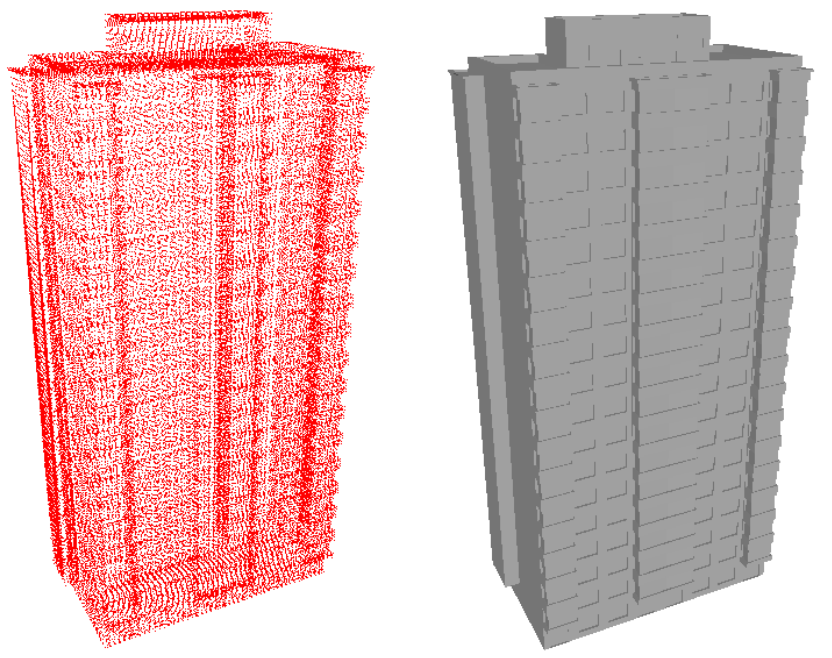

Figure 1. High-resolution geometric building model construction with Lidar data.

For 3D geometric modeling of buildings, it is proposed to extract the façades from point cloud firstly (Yang et al. 2012), and make reasonable organization of them according to some rules (Nan et al. 2010, Furukawa et al. 2010).

\subsection{Structural part extraction}

Structure is the main characteristics that distinguish man-made objects from natural objects, which reflect the relationships between compositional parts of objects. Since the semantic information always manifest as special patterns in the physical world, it is believed that there're inherent connections between geometric structures and semantics. Thus, structural part extraction is the key of semantic 3D building model construction.

As to the structural part recognition of 3D building models, we consider both the macro level parts and detailed parts. The macro level parts are the overall components of building models (Sun et al. 2011a, b), such as the roofs, annex, and different floors, whereas the detailed parts are the basic components of building models (Li et al. 2013), such as the balconies, windows, doors, and chimneys.

- Macro level structural part extraction: volume decomposition algorithms are employed to partition the building model in 3D space, so that the overall compositional parts of the model can be recognized (see Fig. 2 for example).
- Detailed structural part extraction: surface segmentation or geometric analysis algorithms are employed to extract specific features from the outer surface of the building model, so that the tiny visible features can be recognized (see Fig. 3 for example).
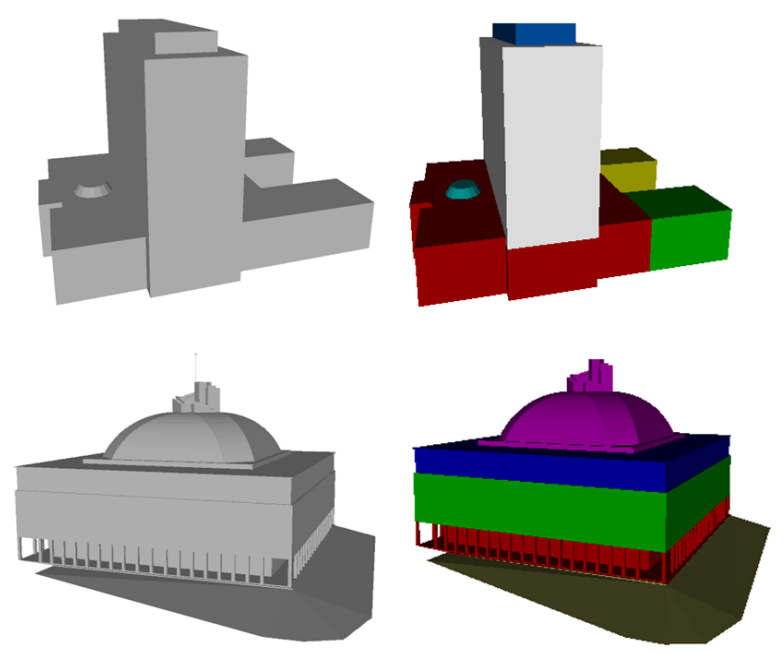

Figure 2. Macro level structural part recognition for 3D building models.
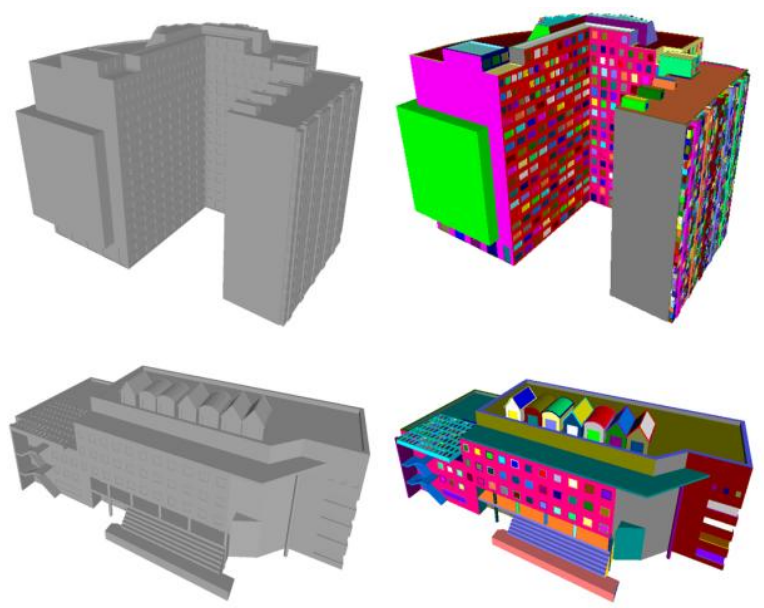

Figure 3. Detailed structural part recognition for 3D building models.

\subsection{Semantic modeling}

After the structural part recognition, semantic 3D building model can be constructed, where the semantic information are organized hierarchically (Zhu \& Hu 2008). The top level records the spatial composition of the building, whereas the bottommost level records all the functional parts. The relationship between higher semantic parts and lower ones is built according to the geometric ownership between them (see Fig. 4). 


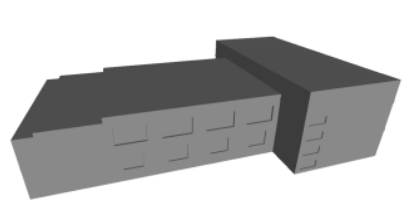

(a)

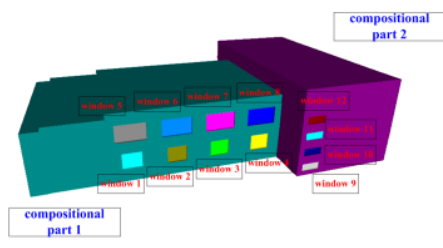

(b)

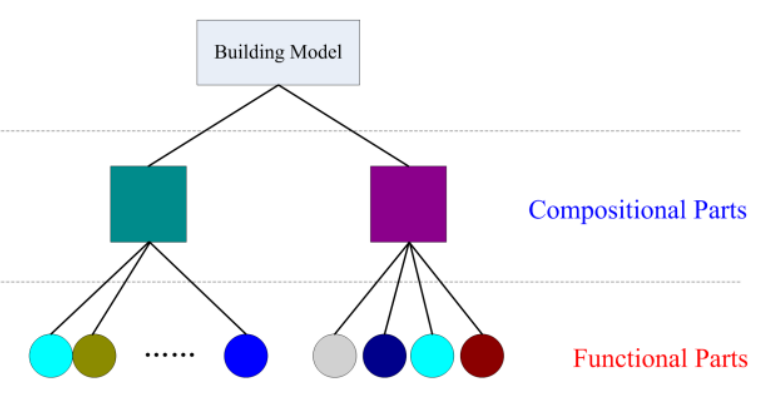

(c)

Figure 4. Semantic 3D building model construction.

\section{EXAMPLE APPLICATIONS}

In modern urban management, the application of semantic 3D building models can greatly improve the efficiency of works in different aspects.

\subsection{Fine-grained spatial analysis}

Spatial analysis is the basis of various smart applications and one of the most important functions of digitized urban management. The capability of it depends on the granularity of spatial data organization, which directly determines how the information needed is integrated.

In traditional 3D spatial analysis, the granularity of spatial data organization is the building model, which means that only the knowledge related to the whole building can be discovered. However, with the aid of semantic building models, the granularity of spatial analysis can be greatly improved. And the knowledge related to different building floors or even different apartments is able to be discovered, which satisfies the increasing sophisticated need of urban management (see Fig. 5).

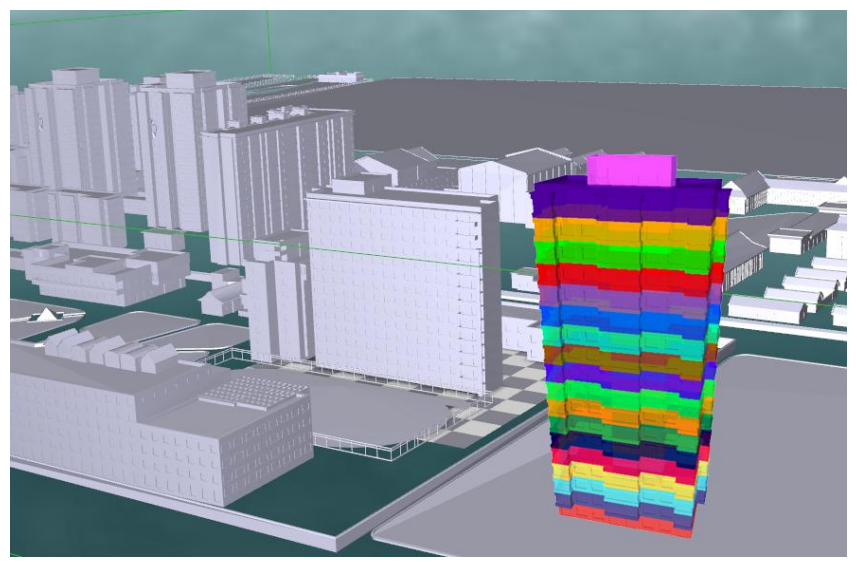

Figure 5. Spatial analysis in the granularity of building floor.

\subsection{Visual estate management}

In the traditional work of estate management, the housing space representation was based on 2D cadastral map and the design papers for building construction. The former reflects the positions and space ranges of buildings in the physical world, whereas the latter describes the inner space structures of each building. Since there is not direct connection between the outer and inner space of buildings, it is hard to determine the accurate position of each apartment from the global coordinate system, which greatly hinder the applications of public security and emergency management in smart city.

With the aid of semantic 3D building models, we can easily distinguish every building apartment from the others in the global urban view, and make visual queries for the information of each apartment and its neighbors on the same or different floor (see Fig. 6).

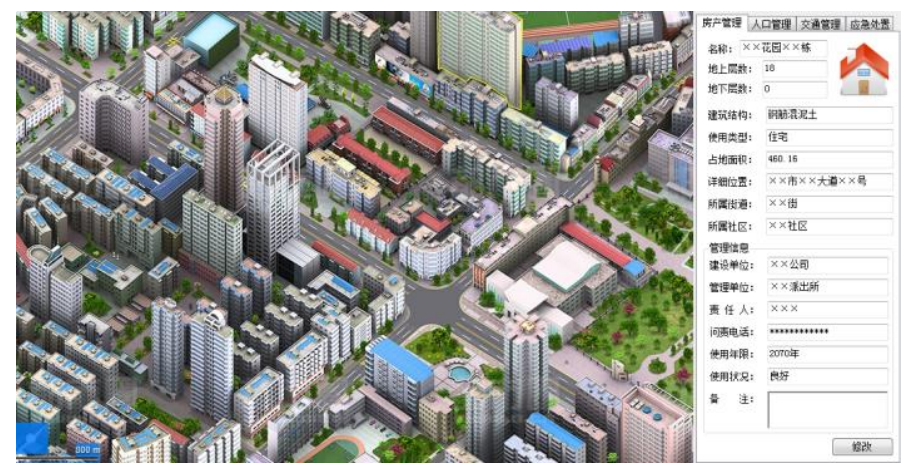

Figure 6. Visual estate management by urban GIS.

\subsection{Informationalized population management}

Since the distribution of population has direct impact on the formation of city, it is of great importance to make efficient population management. However, given the flexible mobility of people, population management has always been a headache to a number of urban governments, especially the ones of big cities. Although lots of measures (e.g. the application of work permit, temporary residential license, and resident permit) have been tested and applied, it is still impossible to give accurate figures about how many residents are living and working in certain area of a city.

On the background of smart city construction, some new ideas are proposed out, such as population management based on location or estate information. With the aid of semantic 3D building models, we can easily bind the information of people with that of the estates (see Fig. 7). Through the integrated information management system, we can not only figure out how many people are there in certain area of the city, but also know the accurate place (e.g. the building number and floor) and environment where each people actually live in. 


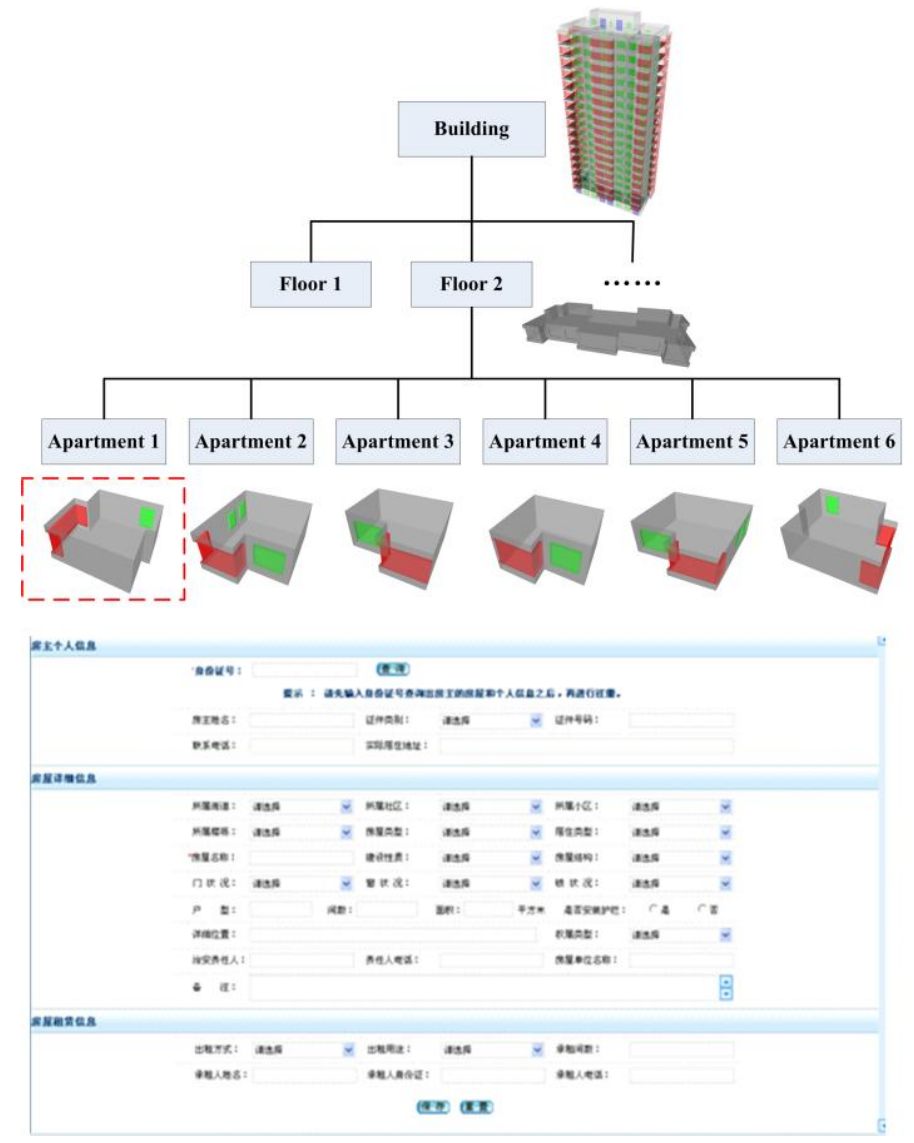

Figure 7. Population management based on estate information.

\section{CONCLUSION REMARKS}

As the basic data of various applications, semantic models are essential to smart urban management. In this paper, we introduced a general and effective scheme to construct semantic 3D building models and illustrated some example applications afterwards.

For widespread usage of semantic 3D building models, there is still much work ahead. As to the model construction process, automatic or semiautomatic algorithms are needed to recognize geometric structures of 3D building models for structural part extraction. And as to the practical applications, the technical issues on data sharing and network transmission are also the obstacles that need to be settled.

\section{REFRENCES}

[1] Benner, J., Geiger, A. \& Leinemann, K. 2005. Flexible generation of semantic 3D building models. In: Proceedings of the 1st international workshop on next generation 3D city models, Bonn, 17-22.

[2] Cerovsek, T. 2011. Advanced Engineering Informatics A review and outlook for a 'Building Information Model' (BIM): A multi-standpoint framework for technological development. Advanced Engineering Informatics 25(2): 224-244.

[3] Elberink, S. \& Vosselman, G. 2009. Building reconstruction by target based graph matching on incomplete laser data: analysis and limitations. Sensors 9: 6101-6118.

[4] Furukawa, Y., Curless, B., Seitz, S. \& Szeliski, R. 2010. Towards Internet-scale Multi-view Stereo. In: IEEE Conference on Computer Vision and Pattern Recognition (CVPR). San Francisco, USA, 1434-1441.

[5] Goetz, M. \& Zipf A. 2012. Towards defining a framework for the automatic derivation of 3D CityGML models from volunteered geographic information. International Journal of 3-D Information Modeling 1(2): 1-16.

[6] Goetz, M. 2013. Towards generating highly detailed 3D CityGML models from OpenStreetMap. International Journal of Geographical Information Science 27(5): 845865.

[7] Gröger, G. \& Plümer, L. 2012. CityGML-Interoperable semantic 3D city models. ISPRS Journal of Photogrammetry and Remote Sensing 71: 12-33.

[8] Isikdag, U. \& Zlatanova, S. 2009. Towards defining a framework for automatic generation of buildings in CityGML using building Information Models. In: Lee J., Zlatanova S. (eds) 3D Geoinformation and Sciences, Springer, Berlin, Heidelberg, 79-96.

[9] Isikdag, U., Zlatanova, S. \& Underwood J. 2013. A BIMOriented Model for supporting indoor navigation requirements. Computers, Environment and Urban Systems 41: 112-123.

[10] Kelly, T. \& Wonka, P. 2011. Interactive Architectural Modeling with Procedural Extrusions. ACM Transactions on Graphics 30(2): 1-15.

[11] Kolbe, H., Gröger, G. \& Plümer, L. 2005. CityGML: Interoperable access to 3D city models. In: Oosterom P., Zlatanova S., Fendel E. (Eds.) Geo-information for Desaster Management. Springer, Berlin, Heidelberg, 883899.

[12] Kolbe, H. 2009. Representing and Exchanging 3D City Models with CityGML. In: Lee J., Zlatanova S. (Eds.) 3D geo-information sciences, Springer, Berlin, Heidelberg, 15-31.

[13] Krecklau, L. \& Kobbelt, L. 2012. Interactive modeling by procedural high-level primitives. Computers \& Graphics 36(5): 376-386.

[14] Li, Q., Sun, X., Yang, B., Jiang, S. 2013. Geometric Structure simplification of 3D building models. ISPRS Journal of Photogrametry and Remote Sensing 84: 100113.

[15] Nan, L., Sharf, A., Zhang, H., Cohen-Or, D. \& Chen B. 2010. SmartBoxes for Interactive Urban Reconstruction. ACM Transactions on Graphics (SIGGRAPH) 29(4): 111.

[16] Sun, X., Yang, B. \& Li, Q. 2011a. Structural Segmentation Method for 3D Building Models Based on Voxel. Acta Geodaetica et Cartographica Sinica 40(5): 582-586.

[17] Sun, X., Yang, B., Attene, M., Li, Q. \& Jiang, S. 2011b. Automated Abstraction of Building Models for 3D Navigation on Mobile Devices. The 19th International Conference on Geoinformatics, June 24-26, Shanghai, China.

[18] Wu, I. \& Hsieh, S. 2007. Transformation from IFC data model to GML data model: methodology and tool development. Journal of the Chinese Institute of Engineers 30(6): 1085-1090.

[19] Yang, B., Wei, Z., Li, Q. \& Li, J. 2012. International Journal of Remote Automated extraction of street-scene objects from mobile lidar point clouds. International Journal of Remote Sensing 33(18): 5839-5861.

[20] Zhu, Q. \& Hu, M. 2008. Semantics-based LOD Models of 3D House Property. Acta Geodaetica et Cartographica Sinica 37(4): 514-520. 